\title{
ON THE HOMOMORPHISM OF VON NEUMANN ALGEBRA
}

\author{
HideO TAKEMOTO
}

(Received November 4, 1968)

1. Introduction. The purpose of this paper is to study the $\sigma$-weak continuity of homomorphism of von Neumann algebra. For the $\sigma$-weak continuity of homomorphism of von Neumann algebra, we have many results that have been shown recently. In this paper, we shall show the following:

Let $M$ be a properly infinite von Neumann algebra with the separable predual $M_{*}$, then any homomorphism $\pi$ from $M$ onto a von Neumann algebra $N$ is $\sigma$-weakly continuous.

Moreover, we shall show some results by using the above fact. Before going into the discussions, the author wishes to express his hearty to Prof. M. Fukamiya and Prof. M. Takesaki in the presentation of this paper.

2. Main result. We start with the following lemma in [8] (Theorem 7 in [8]). In this paper, we shall give a simple proof of Theorem 7 in [8].

LEMMA 1. Let $M$ be a $\sigma$-finite, properly infinite von Neumann algebra, then any *-homomorphism $\pi$ from $M$ into a $\sigma$-finite von Neumann algebra $N$ is $\sigma$-weakly continuous.

PROOF. From Theorem 5 in [8], we have the following decomposition of $\pi ; \pi=\pi_{1} \oplus \pi_{2}$ where $\pi_{1}$ is the $\sigma$-weakly continuous part of $\pi$ and $\pi_{2}$ is the singular part of $\pi$. If $\pi$ is not $\sigma$-weakly continuous, then the singular part $\pi_{2}$ of $\pi$ is not zero. Therefore, we assume that $\pi$ is singular and derive a contradiction. Since $\pi$ is singular, there exists a normal faithful positive linear functional $\psi$ on $N$ such that ${ }^{t} \pi(\psi)=\phi$ is a singular positive linear functional on $M$. Then, from the characterization of a singular positive linear functional in [9] and the $\sigma$-finiteness of $M$, we can find a family $\left\{e_{n}\right\}_{n=1}^{\infty}$ of countable orthogonal projections in $M$ such that $1=\sum_{n=1}^{\infty} e_{n}$ and $\phi\left(e_{n}\right)=0$ for all $n$. Since $0=\varphi\left(e_{n}\right)={ }^{t} \pi(\psi)\left(e_{n}\right)=\psi\left(\pi\left(e_{n}\right)\right)$ and $\psi$ is faithful, $\pi\left(e_{n}\right)=0$. 
Therefore, there exists a family $\left\{e_{n}\right\}_{n=1}^{\infty}$ of countable orthogonal projections such that $\sum_{n=1}^{\infty} e_{n}=1$ and $\pi\left(e_{n}\right)=0$ for all $n$.

Now, since $M$ is properly infinite, there exists in $M$ a family $\left\{p_{n}\right\}_{n=1}^{\infty}$ of orthogonal projections such as $p_{n} \sim 1$. Take a partial isometry $v_{n}$ such that $v_{n}^{*} v_{n}=p_{n}$ and $v_{n} v_{n}^{*}=1$ for each $n$. Define the family $\left\{q_{n}\right\}_{n=1}^{\infty}$ of orthogonal projections by $q_{n}=v_{n}^{*}\left(\sum_{k=1}^{n} e_{k}\right) v_{n}$.

Let $\left\{n_{i}\right\}_{i=1}^{\infty}$ be an increasing sequence of positive integers, then we have

$$
q_{n_{t+1}}=v_{n_{t+1}}^{*}\left(\sum_{k=1}^{n_{t+1}} e_{k}\right) v_{n_{t+1}} \geqq v_{n_{t+1}}^{*}\left(\sum_{k=n_{t+1}}^{n_{t+1}} \boldsymbol{e}_{k}\right) v_{n_{t+1}} \sim \sum_{k=n_{t}+1}^{n_{t+1}} \boldsymbol{e}_{k}
$$

Hence $\sum_{i=1}^{\infty} q_{n_{\iota}} \succsim \sum_{k=n_{1}+1}^{\infty} e_{k}$, and $\pi\left(\sum_{i=1}^{\infty} q_{n_{t}}\right) \succsim \pi\left(\sum_{k=n_{1}+1}^{\infty} e_{k}\right)=\pi\left(1-\sum_{k=1}^{n_{1}} e_{k}\right)=\pi(1) \neq 0$. Therefore $\pi\left(\sum_{i=1}^{\infty} q_{n_{i}}\right) \neq 0$. On the other hand $\pi\left(q_{n}\right)=\pi\left(v_{n}\right) *\left(\sum_{k=1}^{n} \pi\left(e_{k}\right)\right) \pi\left(v_{n}\right)=0$.

Next, let $\left\{r_{n}\right\}_{n=1}^{\infty}$ be a countable set of all rational numbers. For each real number $s$, we can choose an infinite subsequence $\left\{r_{n_{1}}\right\}_{i=1}^{\infty}$ of $\left\{r_{n}\right\}_{n=1}^{\infty}$ such that, for each positive integer $i$, we have $0<\left|r_{n_{i}}-s\right|<1 / i$ and $n_{j}<n_{i}$ for every $j<i$. Now, let us correspond to $s$ the index set $\left\{n_{i}\right\}_{i=1}^{\infty}$ of the above sequence $\left\{r_{n_{i}}\right\}_{i=1}^{\infty}$. Then if $s \neq s^{\prime}$ for real numbers $s$ and $s^{\prime},\left\{n_{i}\right\} \cap\left\{n_{i}^{\prime}\right\}$ is at most a finite set where $\left\{n_{i}\right\}$ and $\left\{n_{i}^{\prime}\right\}$ are corresponding index sets of $s$ and $s^{\prime}$ respectively, because $\left\{r_{n_{1}}\right\}_{i=1}^{\infty}$ and $\left\{r_{n^{\prime}}\right\}_{i=1}^{\infty}$ converge to $s$ and $s^{\prime}$ respectively. Therefore, if we set $q_{s}=\sum_{i=1}^{\infty} q_{n_{t}}$ for a real number $s$ where $\left\{n_{i}\right\}_{i=1}^{\infty}$ is an index set corresponding to $s$, we get $\pi\left(q_{s}\right) \neq 0$ and $\pi\left(q_{s} q_{s^{\prime}}\right)=0$ if $s \neq s$. Hence the family $\left\{\pi\left(q_{s}\right) ; s \in R\right\}$ where $R$ is the set of all real numbers is a family of orthogonal non-zero projections of $N$ with the power of continuum c. But, $N$ is $\sigma$-finite, this is a contradiction. Therefore, $\pi$ does not admit the singular part, hence $\pi$ is $\sigma$-weakly continuous. This completes the proof of Lemma 1.

Next, we shall need a result due to T. Okayasu [4].

Lemma 2. (The Polar Decomposition Theorem). Let $A$ be a $C^{*}$-algebra with the identity 1 , then any isomorphism $\pi$ (not necessary *-preserving) from $A$ onto a von Neumann algebra $M$ has the decomposition: $\pi=\pi_{1} \circ \pi_{2}$, where $\pi_{1}$ is an inner automorphism of $M$ and $\pi_{2}$ is a $*_{\text {-isomorphism from }}$ A onto $M$. 
By Lemma 2, we have

LEMMA 3. Let $M$ and $N$ be von Neumann algebras acting on Hilbert spaces $H$ and $K$ respectively. If any *-homomorphism from $M$ onto $N$ is $\sigma$-weakly continuous, then any homomorphism $\pi$ from $M$ onto $N$ is $\sigma$-weakly continuous.

ProOF. Since $\pi(M)=N$, by the Rickart's theorem, the kernel $\pi^{-1}(0)$ of $\pi$ is a uniformly closed two-sided ideal, hence the quotient algeb:a $M / \pi^{-1}(0)$ is a $C^{*}$-algebra. Let $\delta$ be the canonical mapping from $M$ onto $M / \pi^{-1}(0)$, then $\delta$ is a *-homomorphism. Let $\tilde{\pi}$ be the mapping from $M / \pi^{-1}(0)$ onto $N$ which is induced canonically by $\pi$, then $\tilde{\pi}$ is an isomorphism from $M / \pi^{-1}(0)$ onto $N$. Therefore, by Lemma 2, we have the following decomposition of $\tilde{\pi}: \tilde{\pi}=\tilde{\pi}_{1} \circ \tilde{\pi}_{2}$ where $\tilde{\pi}_{1}$ is an inner automorphism of $N$ and $\tilde{\pi}_{2}$ is a *-isomorphism from $M / \pi^{-1}(0)$ onto $N$, whence we have : $\pi=\widetilde{\pi} \circ \delta=\widetilde{\pi}_{1} \circ\left(\tilde{\pi}_{2} \circ \delta\right)$. Since $\tilde{\pi}_{1}$ is the inner automorphism of $N$, it is $\sigma$-weakly continuous. Furthermore, since, by the assumption, $\tilde{\pi}_{2} \circ \delta$ is a ${ }^{*}$-homomorphism from $M$ onto $N$, it is $\sigma$-weakly continuous. Therefore, $\pi$ is $\sigma$-weakly continuous. This completes the proof of Lemma 3.

COROLlARY. Let $M$ be a $\sigma$-finite, properly infinite von Neumann algebra, then any homomorphism $\pi$ from $M$ onto a $\sigma$-finite von Neumann algebra $N$ is $\sigma$-weakly continuous.

By using the above results, we can show the main result.

THEOREM I. Let $M$ be a properly infinite von Neumann algebra acting on a Hilbert space $H$ and having the separable predual $M_{*}$, then any homomorphism $\pi$ from $M$ onto a von Neumann algebra acting on a Hilbert space $K$ is $\sigma$-weakly continuous.

PROOF. To prove Theorem I, we may suppose, by Lemma 1 and Lemma 3 , that $\pi$ is a ${ }^{*}$-homomorphism. At first, we shall show that the cardinal number of the set of all projections in $M$ is not larger than c. For each non-zero projection $e$ in $M$, there exists a normal state $\psi$ on $M$ such that $\operatorname{supp}(\psi) \leqq e$, and so, for any family $\left\{e_{\lambda}\right\}_{\lambda \in A}$ of mutually orthogonal projections in $M$, there exists a family $\left\{\psi_{\lambda}\right\}_{\lambda_{\epsilon} A}$ of normal states on $M$ such that $\operatorname{supp}\left(\psi_{\lambda}\right) \leqq e_{\lambda}$ for each $\lambda \in \Lambda$. Since $\left\{e_{\lambda}\right\}_{\lambda \in \Lambda}$ is mutually orthogonal, we have: if $\lambda, \mu \in \Lambda$ and $\lambda \neq \mu,\left\|\psi_{\lambda}-\psi_{\mu}\right\|=\left\|\psi_{\lambda}\right\|+\left\|\psi_{\mu}\right\|=2$. Let $\left\{\varphi_{n}\right\}_{n=1}^{\infty}$ be a countable dense subset of $M_{*}$, then, for each $\lambda \in \Lambda$, there exists an element $\boldsymbol{\phi}_{n_{\lambda}}$ in $\left\{\boldsymbol{\phi}_{n}\right\}_{n=1}^{\infty}$ with $\left\|\psi_{\lambda}-\boldsymbol{\phi}_{n_{\lambda}}\right\|<1 / 2$. Furthermore, for two distinct $\lambda$ and $\mu$ of $\Lambda, \phi_{n_{\lambda}} \neq \phi_{n_{\mu}}$. Therefore, we see that any family of mutually orthogonal 
non-zero projections in $M$ is at most countable and $M$ is a $\sigma$-finite von Neumann algebra. Since $M$ is $\sigma$-finite, for each non-zero projection $e$ in $M$, there exists a normal state $\psi_{e}$ on $M$ with $\operatorname{supp}\left(\psi_{e}\right)=e$, and it is clear that, for two distinct projections $e$ and $f$ in $M, \psi_{e} \neq \psi_{f}$. That is, the cardinal number of the set of all projections in $M$ is not larger than the cardinal number of $M_{*}$. Now, since $M_{*}$ is separable, the cardinal number of $M_{*}$ is c. Therefore, the cardinal number of the set of all projections in $M$ is not larger than $\mathrm{c}$.

Next, we shall show that $N$ is a $\sigma$-finite von Neumann algebra. If otherwise, the cardinal number of the set of all projections in $N$ will be exactly larger than c. In fact, we can take a family $\left\{\boldsymbol{e}_{\alpha}\right\}_{\alpha \in A}$ of orthogonal projections in $N$ where $A$ is the index set with the cardinal number $\beta$ which is exactly larger than $\boldsymbol{N}_{0}$, because $N$ is supposed not to be $\sigma$-finite. Then, the cardinal number of the set of all subsets of $\left\{\boldsymbol{e}_{\alpha}\right\}_{\alpha \in A}$ is exactly larger than $\mathfrak{c}$, and so the cardinal number of the set of all projections in $N$ will be exactly larger than c. Therefore, if $N$ is not a $\sigma$-finite von Neumann algebra, then the cardinal number of the set of all projections in $N$ is exactly larger than the continuum cardinal number c. But, for each projection $p$ in $N$, there exists a projection $e$ in $M$ such that $\pi(e)=p$ (Theorem 3.2 in [10]). As we have proved above, the cardinal number of the set of all projections in $M$ is not larger than the continuum cardinal number $\mathfrak{c}$. Therefore, we have a contradiction, and so $N$ is a $\sigma$-finite von Neumann algebra.

By the above argument, $M$ and $N$ are $\sigma$-finite von Neumann algebras. Therefore, by Lemma 1 , if $\pi$ is any *-homomorphism from $M$ onto $N$, then $\pi$ is $\sigma$-weakly continuous. Furthermore, by Corollary of Lemma 3, even if $\pi$ is not ${ }^{*}$-preserving, $\pi$ is $\sigma$-weakly continuous. This completes the proof of Theorem I.

REMARK 1. The above result has some applications in the reduction theory ([7]). Besides we can show the following Theorem II.

Following the notation in J. Glimm [3], let $M$ be a von Neumann algebra, $Z$ be the center of $M$ and $X$ be the spectrum of $Z$. If $\zeta \in X$, let $I(\zeta)$ be the uniform closure of the set $\left\{\sum_{i=1}^{n} z_{i} a_{i} ; n\right.$ is a positive integer, $a_{i}$ is an element of $M$ and $z_{i}$ is a central element with $z_{i}{ }^{\wedge}(\zeta)=0 !$ where $z_{i}{ }^{\wedge}$ is the Gelfand representation of $z_{i}$. Then $I(\zeta)$ is a uniformly closed two-sided ideal in $M$, and the quotient algebra $M(\zeta)=M / I(\zeta)$ is a $C^{*}$-algebra. However, it has not yet been certain whether $M(\zeta)$ is a von Neumann algebra or not. In the remaning part, we shall show that $M(\zeta)$ is not necessary a von 
Neumann algebra.

Let $\pi_{\zeta}$ be the canonical mapping from $M$ onto $M(\zeta)$. Then, for $\zeta \in X$, $\pi_{\zeta}^{-1}(0) \cap Z=\left\{z \in Z ; z^{\wedge}(\zeta)=0\right\}$ where $\pi_{\zeta}^{-1}(0)$ is the kernel of $\pi_{\zeta}$. Therefore, $\pi_{\zeta}$ is $\sigma$-weakly continuous if and only if the set $\{\zeta\}$ is a closed and open set in $X$, and $\pi_{\zeta}$ is $\sigma$-weakly continuous for all $\zeta \in X$ if and only if $Z$ is an atomic abelian von Neumann algebra. By considering the above argument, we have the following result.

THEOREM II. Let $M$ be a properly infinite von Neumann algebra with the separable predual $M_{*}$ and the center $Z$ wich is non-atomic. Then there exists an element $\zeta$ of the spectrum $X$ of $Z$ for which $M(\zeta)$ is not a von Neumann algebra.

PROOF. Since $Z$ is non-atomic, there exists an element $\zeta$ of $X$ such that $\pi_{\zeta}$ is not $\sigma$-weakly continuous. If $M(\zeta)$ is a von Neumann algebra for such an element $\zeta$, then $\pi_{\zeta}$ is a ${ }^{*}$-homomorphism from $M$ onto a von Neumann algebra $M(\zeta)$, and, by Theorem I, $\pi_{\zeta}$ must be $\sigma$-weakly continuous; this is a contradiction. Therefore, $M(\zeta)$ can't be a von Neumann algebra, which completes the proof.

We may construct a von Neumann algebra which satisfies the condition in Theorem II. Let $H$ be a countably infinite dimensional Hilbert space, $B(H)$ the von Neumann algebra of all bounded operators on $H$ and $L^{\infty}(0,1)$ a von Neumann algebra of all essentially bounded functions under the Lebesgue measure $\mu$ on the open interval $(0,1)$. As $B(H)$ is properly infinite and $L^{\infty}(0,1)$ is a finite von Neumann algebra, the $W^{*}$-tensor product $M=L^{\infty}(0,1) \otimes B(H)$ is a properly infinite von Neumann algebra (p. 3.40 in [6]) and the center $Z$ of $M$ is $L^{\infty}(0,1) \otimes C_{H}$ where $\boldsymbol{C}_{H}$ is the von Neumann algebra of all scalar multiples of the identity operator on $H$. Therefore, $Z$ is a non-atomic abelian von Neumann algebra. Since the Hilbert space $L^{2}(0,1) \otimes H$ is separable, the predual $M_{*}$ of $M$ is separable.

REMARK 2. Prof. M. Takesaki kindly communicated me that Theorem I in the present paper may be applied to show the statement that: if $H$ is a countably infinite dimensional Hilbert space, then the quotient algebra $B(H) / C(H)$ is not a von Neumann algebra where $B(H)$ is the von Neumann algebra of all bounded operators on $H$ and $C(H)$ is the uniformly closed two-sided ideal of all completely continuous operators on $H$. In fact, $B(H)$ is a properly infinite von Neumann algebra and the canonical mapping $\pi$ from $B(H)$ onto $B(H) / C(H)$ is not $\sigma$-weakly continuous (cf.[11]). 


\section{REFERENCES}

[1] J.W. CALKIN, Two sided ideals and congruences in the rings of bounded operators in Hilbert space, Ann. of Math., 42(1941), 839-873.

[2] J. DIXMIER, Les algébras d'operateurs dans l'espace hilbertien, Gauthier-Villars, Paris, 1957.

[3] J. Glimm, The Stone-Weierstrass Theorem for $C^{*}$-algebras, Ann. of Math., 72(1960), 216-244.

[4] T. OKAYASU, A structure theorem of automorphism of von Neumann algebras, Tôhoku Math. J., 20(1968), 199-206.

[5] C. RICKART, General theory of Banach algebras, Van Nostrand, New York, 1960.

[6] S. SAKAI, The theory of $W^{*}$-algebras, Lecture Note, Yale university, 1962.

[7] H. TAKEMOTO, On the integral representation of some functional on a von Neumann algebra, to appear in Tôhoku Math. J., 21(1969).

[8] M. TAKESAKI, On the conjugate space of operator algebra, Tôhoku Math. J., 10(1958), 194-203.

[9] M. TAKESAKI, Singularity of positive linear functionals, Proc. Japan Acad., 36(1959), 365-366.

[10] F. B. WRIGHT, A reduction for algebras of finite type, Ann. of Math., 60(1954), 560-570.

[11] I. KAPLANSKY, Representations of separable algebras, Duke Math. J., 19 (1952), 219-222.

MATHEMATICAL INSTITUTE

TÔHOKU UNIVERSITY

SENDAI, JAPAN 